\title{
ASOCIACIÓN ENTRE INFECCIÓN LEPTOSPIRAL Y PROBLEMAS REPRODUCTIVOS EN OVEJAS DE UNA EMPRESA GANADERA EN LA SIERRA CENTRAL DEL PERÚ
}

\author{
Association of Leptospira Infection and Reproductive Problems in a Large \\ Sheep F arm in the Central Highlando of Peru
}

Andrea Flores M. ${ }^{1}$, Hermelinda Rivera G. ${ }^{1,2}$ y César Gavidia Ch. ${ }^{3}$

\section{ResUMen}

\begin{abstract}
El objetivo del presente estudio fue determinar la asociación entre la infección por Leptospira sp. y la presentación de problemas reproductivos en ovejas de dos localidades de una Sociedad Agrícola de Interés Social (SAIS), en Junín, durante la campaña reproductiva del 2003, a través de un estudio de Caso-Control. Se colectaron muestras de suero de ovejas entre 2 a 10 años de edad que abortaron o que no quedaron gestantes después de uno o dos empadres (grupo Caso: $n=220$ ) y de borregas sin problemas reproductivos (grupo Control: $\mathrm{n}=220$ ) para determinar anticuerpos contra Leptospira, serovares pomona, hardjo, canicola, icterohaemorrhagiae, grippotyphosa, wolffi, ballum y bratislava, mediante la técnica de microaglutinación. La frecuencia de Leptospira sp. fue de $24.8 \pm 4.6 \%$, correspondiendo al grupo Caso $28.6 \pm 7.0 \%$ y al grupo Control 20.9 $\pm 5.9 \%$. No se encontró asociación estadística entre las variables presencia de anticuerpos y problemas reproductivos mediante el análisis de Chi Cuadrado. Los serovares detectados con mayor frecuencia fueron ballum con $42.2 \%$ (46/109) seguido por icterohaemorrhagiae con $31.2 \%$ (34/109). Se detectó animales con anticuerpos contra más de un serovar de Leptospira.
\end{abstract}

Palabras clave: ovinos, borregas, Leptospira sp., abortos, anticuerpos, microaglutinación

\section{Abstract}

The association between leptospiral infection and reproductive problems in ewes from a large sheep enterprise in Junin, Peru, was evaluated in a Case-Control study during the breeding season of 2003. Serum samples from 2-10 year-old ewes with abortion or infertility problems (Case group, $\mathrm{n}=220$ ) and from ewes without reproductive problems (Control group, $\mathrm{n}=220$ ) were collected for the detection of antibodies against Leptospira serovar: pomona, hardjo, canicola, icterohaemorrhagiae, grippotyphosa, wolffi, ballum and bratislava by the microscopic agglutination test. The prevalence of Leptospira sp. was $24.8 \pm 4.6 \%$, corresponding $28.6 \pm 7.0 \%$ to the Case group and $20.9 \pm 5.9 \%$ to the

\footnotetext{
${ }^{1}$ Laboratorio de Microbiología y Parasitología Veterinaria, ${ }^{3}$ Laboratorio de Medicina Veterinaria Preventiva, Facultad de Medicina Veterinaria, Universidad Nacional Mayor de San Marcos, Lima

${ }^{2}$ E-mail: hriverag2005@yahoo.es
} 
Control group. No statistic association between leptospiral antibodies and reproductive problems was found by the Chi Square test. The highest serological prevalence $(42.2 \%)$ was recorded for serovar ballum followed by icterohaemorrhagiae (31.2\%). Animals with antibodies to more than one serovar of Leptospira were detected.

Key words: sheep, ewe, Leptospira sp., abortion, antibodies, microscopic agglutination test

\section{INTRODUCCIÓN}

La población ovina en el Perú es, aproximadamente, de 12085683 cabezas (INEI, 1994), distribuyéndose mayormente en la sierra, seguido de la costa y la selva (DGIA, 2002). El $70 \%$ de esta población pertenece a comunidades campesinas y criada en sistemas de tipo extensivo y mixto, con escasa o ninguna tecnología. Sin embargo, en la Sierra Central existen dos empresas campesinas o SAIS (Sociedad Agrícola de Interés Social) que han logrado un aceptable nivel tecnológico y rebaños entre 80000 y 120000 cabezas que les permite manejar una ganadería más rentable (DGIA, 2002).

Los ovinos, como otros rumiantes domésticos, son afectados por agentes infecciosos que les ocasionan abortos, mortalidad perinatal, etc. Dentro de los agentes bacterianos causantes de estos problemas se encuentra la Leptospira sp. (Schoenian, 2000), que son bacterias de amplia distribución mundial y de compleja epidemiología (Liceras, 1989). Estudios de prevalencia en humanos, así como en algunas especies de animales domésticos y silvestres en el Perú, confirman su difusión en el país (Liceras, 1989).

El aborto y, en general, los problemas reproductivos, ocasionan pérdidas económicas para el ganadero y son de difícil diagnóstico debido a su variada etiología. En el ovino, a diferencia del bovino, donde la ocurrencia del aborto y otros problemas reproductivos superan el 10\% (Rivera, 2001), el aborto constituye el $6 \%$; pero en este caso, donde se tra- ta de miles de animales que conforman un rebaño, las pérdidas económicas son cuantiosas para la empresa o el criador (Gamarra M, comunicación personal).

No se dispone de información sobre el rol de las Leptospira en la presentación de los problemas reproductivos en ovinos del país, por lo que el objetivo del presente estudio fue determinar la asociación entre la presenciace antiarenos contraLeptospira sp. y los problemas reproductivos en borregas de una SAIS durante la campaña reproductiva del 2003.

\section{Materiales y Métodos}

El estudio se realizó en dos zonas (I y II) de pastoreo de una Sociedad Agrícola de Interés Social (SAIS), ubicada entre 3500 a 4700 msnm, en Huancayo, Junín. Se trabajó con borregas de raza Junín de entre 2 a 10 años de edad durante la faena del perneo, que es el diagnóstico de la preñez mediante el baloteo del vientre y la marcación de los corderos, actividad que se realiza en noviembre de cada año.

Las borregas que fueron diagnosticadas como vacías después del primer empadre (vacías $\mathrm{x}$ 1) y que tampoco preñaron en el segundo empadre (vacías x 2) y aquellas que abortaron constituyeron el grupo CASO $(\mathrm{n}=$ 220) mientras que el grupo CONTROL $(\mathrm{n}=$ 220) estuvo constituido por borregas que no tuvieron problemas reproductivos durante esa campaña reproductiva (año 2003). 
Se obtuvo muestras de sangre de la vena yugular o safena con agujas y tubos vacutainers. El suero fue obtenido por centrifugación y transportado al Laboratorio de Microbiología y Parasitología Veterinaria de la Facultad de Medicina Veterinaria de la Universidad Nacional Mayor de San Marcos, en Lima, para su procesamiento.

Se evaluó la presencia de anticuerpos contra los siguientes serovares de Leptospira: pomona, hardjo, canicola, icterohaemorrhagiae, grippotyphosa, wolffi, ballum y bratislava, mediante la prueba de Microaglutinación (MAT) empleando antígeno vivo de dichos serovares y de acuerdo al protocolo descrito en el Manual de Leptospirosis de la Asociación Argentina de Veterinarios de Laboratorios de Diagnóstico (AAVLD, 1994). Una muestra fue considerada positiva a anticuerpos contra Leptospira cuando al ser diluida en 1:100, fue capaz de aglutinar al 50\% ó más de las leptospiras presentes en el campo de observación del microscopio de campo oscuro.

La asociación entre la seropositividad a Leptospira y presentación de problemas reproductivos en las borregas se analizó mediante la prueba de Chi Cuadrado y el software STATA ${ }^{\circledR}$.

\section{Resultados y Discusión}

La frecuencia de Leptospira sp. en la población de borregas en estudio fue de 24.8 $\pm 4.5 \%$ (109/440), correspondiendo al grupo CASO $28.6 \pm 7.0 \%(63 / 220)$ y al grupo CONTROL $20.9 \pm 5.9 \%$ (46/220). No se encontró significancia estadística entre las variables presencia de anticuerpos y ocurrencia de problemas reproductivos. Asimismo, las borregas con problemas reproductivos de ambas zonas de pastoreo presentaron similares porcentajes de animales serorreactores a Leptospira sp. (Cuadro 1).
Se encontró una frecuencia de anticuerpos contra 6 de 8 serovares de Leptospira sp. de $24.8 \pm 4.5 \%$ (109/440) en borregas con o sin problemas reproductivos. Esto es una evidencia de infecciones naturales de Leptospira, ya que en el calendario sanitario que utiliza la SAIS en estudio no figura la vacunación contra esta bacteria.

La presencia de anticuerpos contra los serovares de Leptospira pomona, icterohaemorrhagiae, grippotyphosa, ballum y wolffi (Cuadro 3), indica que estos agentes están distribuidos en la zona y que existen condiciones ambientales óptimas como humedad, ojos de agua, suelo neutro a ligeramente alcalino, etc., y de la presencia de hospedadores de mantenimiento que pueden ser roedores $\mathrm{u}$ otros animales silvestres o domésticos en los cuales la infección es de tipo subclínico, pero con eliminación de la bacteria por la orina contaminando el agua y los pastos (Bolin y Alt, 1998; Vanasco et al., 2001).

La falta de asociación entre las variables presencia de anticuerpos y problemas reproductivos durante la campaña del 2003 en las borregas podría deberse a factores medioambientales, compleja epidemiología de la leptospirosis, escasa información sobre la generación y duración de la inmunidad humoral en las borregas frente a este agente, etc. Los resultados serológicos, sin embargo, sugieren que la Leptospira podría ser uno de los agentes involucrados en la presentación del aborto o infertilidad ya que los serovares ballum, icterohaemorrhagiae, pomona y grippotyphosa tienen mayores frecuencias en el grupo CASO, incluso algunos con títulos de anticuerpos de 3200 comparado con el grupo CONTROL (Cuadros 3 y 4). 
Dentro del grupo de animales seropositivos se detectaron animales con anticuerpos hasta contra tres serovares, lo cual implica infecciones mixtas. La asociación entre serovares icterohaemorrhagiae y ballum (14.68\%) fue la más frecuente (Cuadro 2), siendo los hospedadores de mantenimiento de estos serovares los roedores silvestres. También, el mayor número de borregas con infecciones mixtas y con títulos de anticuerpos entre 100 y 800 se observaron en el grupo CASO. No obstante, la presencia de anticuerpos en diluciones bajas como 100 en las borregas con infecciones mixtas, podría deberse a reacciones cruzadas entre algunos serovares, sobre todo durante infecciones agudas; sin embargo, en títulos mayores a 400 , es más probable que se deban a serovar específico (Cuadros 3 y 4). Se menciona que los anticuerpos serovar específico predominan en el animal durante la convalecencia (Awad y Willinger, 1982).

La ausencia de anticuerpos contra los serovares hardjo y bratislava es rara, ya que estos serovares han sido detectados en bovinos y ovinos (Hathaway et al., 1982; Ellis, 1994; Zamora et al., 1999; Ochoa et al., 2000); aunque Blackmore et al. (1979) mencionan que el serovar hardjo, a diferencia del bovino, no es endémico en ovinos, pero puede afectarlos esporádicamente. Los serovares de menor frecuencia fueron canicola y wolffi, ambos con títulos bajos, pudiendo tratarse también de reacciones cruzadas con otros serovares, y solo fueron detectados en el grupo CASO. En un estudio realizado en humanos, bovinos y perros del valle del Mantaro, se reporta anticuerpos contra el serovar canicola (2.8\%) en las tres especies estudiadas, pero solo wolffi $(0.9 \%)$ en bovinos (Céspedes et al., 2003), indicando que ambos serovares están presentes en la Sierra Central pero que sus prevalencias en las zonas de estudio podrían haber sido bajas. El hospedador de mantenimiento del serovar canicola es el perro y en las puntas de las borregas existen perros ovejeros; sin embargo, la baja prevalencia en las zonas de estudio disminuye el riesgo de infección para los perros.

En el país no se dispone de información sobre la presentación clínica de leptospirosis en ovinos, pero la presencia de anticuerpos indica que la bacteria está presente y que la infección sería mayormente de tipo subclínica, o quizás está siendo confundida con otras infecciones. Sin embargo, se debe tener presente que ciertos serovares como pomona pueden ocasionar brotes de leptospirosis con alta mortalidad sobre todo en corderos (Vermunt et al., 1994).

Existen escasos estudios epidemiológicos de Caso-Control de leptospirosis en ovinos que permitan contrastar el presente estudio. Sin embargo, el porcentaje de borregas serorreactoras en ambos grupos es mayor a la prevalencia de $15.3 \%$ reportada en el país por Quiroz (1969) y en otros países como Chile: 5.7\% (Zamora et al., 1999); Bolivia: 14.3\% (Ciceroni et al., 1997); Brasil: 8.6\% (Barbudo Filho et al., 1999); Italia: $6.1 \%$ (Ciceroni et al., 2000); y Nueva Zelanda: $17.1 \%$ (Collings, 1984) entre otros, con excepción del estudio de Herrmann et al. (2004) realizado en Rio Grande do Sul, Brasil, donde se reporta una prevalencia de $34.3 \%$, mayor a la obtenida en el presente estudio.

La prueba de MAT es la técnica de referencia para el diagnóstico de la leptospirosis (OIE, 2006). La prueba puede ser aplicada para detección de anticuerpos en varias especies de animales, incluyendo el hombre, e identifica el serovar infectante pero, a diferencia de la prueba de ELISA, no distingue las clases de inmunoglobulinas IgM o IgG que pudiera indicar infección temprana o tardía (Levett, 2001). No existen datos sobre el grado de sensibilidad y especificidad de la prueba, posiblemente porque depende de varios factores. En un estudio de comparación con la prueba de aglutinación en látex, se indica que la prueba de MAT tiene una sensibilidad de $85 \%$ cuando la infección tiene 30 días o más de haberse iniciado (Smits et al., 2000). 
Cuadro 1. Animales del grupo CASO con anticuerpos contra Leptospira sp. de dos zonas de pastoreo en una SAIS de Huancayo (2003)

\begin{tabular}{lccccc}
\hline & \multirow{2}{*}{$\begin{array}{c}\text { Animales } \\
\text { Tipo de problema }\end{array}$} & \multicolumn{5}{c}{ Animales positivos } \\
\cline { 3 - 6 } reproductivo & $(\mathrm{n})$ & $\mathrm{C}$ & $\mathrm{II}$ & $\mathrm{N}^{\mathrm{o}}$ & $\%$ \\
\cline { 3 - 6 } & 133 & 16 & 18 & 34 & 25.56 \\
\hline Abortos & 34 & 11 & 2 & 13 & 38.24 \\
Vacías x 1 & 53 & 11 & 5 & 16 & 30.19 \\
Vacías x 2 & 220 & 38 & 25 & 63 & 28.64 \\
\hline Total & & & & & \\
\hline
\end{tabular}

Cuadro 2. Ovejas serorreactoras a ocho serovares de Leptospira sp. en una empresa ganadera de la Sierra Central (2003)

\begin{tabular}{lcc}
\hline \multirow{2}{*}{ Serovar de Leptospira } & \multicolumn{2}{c}{ Animales positivos } \\
\cline { 2 - 3 } & $\mathrm{N}^{\mathrm{o}}$ & $\%$ \\
\hline pomona & 5 & 4.6 \\
hardjo & 0 & 0 \\
canicola & 1 & 0.9 \\
icterohaemorrhagiae & 34 & 31.2 \\
grippotyphosa & 3 & 2.8 \\
wolffi & 0 & 0 \\
ballum & 45 & 41.3 \\
bratislava & 0 & 0 \\
icterohaemorrhagiae + ballum & 16 & 14.7 \\
pomona + ballum & 3 & 2.8 \\
wolffi + grippotyphosa & 1 & 0.9 \\
Icterohaemorrhagiae + canicola + ballum & 1 & 0.9 \\
\hline Total & 109 & $100 \%$ \\
\hline
\end{tabular}


Cuadro 3. Animales serorreactores en una empresa ganadera de la Sierra Central y títulos de anticuerpos aglutinantes contra los serovares de Leptospira sp. en el grupo CASO (2003)

\begin{tabular}{|c|c|c|c|c|c|c|c|}
\hline \multirow{2}{*}{ Leptospira sp. } & \multicolumn{2}{|c|}{ Positivos } & \multicolumn{5}{|c|}{ Diluciones del suero } \\
\hline & $\mathrm{N}^{\circ}$ & $\%$ & 100 & 200 & 400 & 800 & 3200 \\
\hline pomona & 5 & 7.9 & 4 & 0 & 0 & 1 & 0 \\
\hline hardjo & 0 & 0 & 0 & 0 & 0 & 0 & 0 \\
\hline canicola & 1 & 1.6 & 1 & & & & \\
\hline icterohaemorrhagiae & 14 & 22.2 & 7 & 7 & 0 & 0 & 0 \\
\hline grippotyphosa & 3 & 4.78 & 1 & 1 & 0 & 1 & 1 \\
\hline wolffi & 0 & 0 & 0 & 0 & 0 & 0 & 0 \\
\hline ballum & 25 & 39.7 & 9 & 10 & 4 & 1 & 1 \\
\hline bratislava & 0 & 0 & 0 & 0 & 0 & 0 & 0 \\
\hline icterohaemorrhagiae + ballum & 10 & 17.5 & $4 i / 4 b$ & $6 i / 3 b$ & $3 b$ & 0 & 0 \\
\hline pomona + ballum & 3 & 4.8 & 1 & $1 p / 3 b$ & 0 & 1 & 0 \\
\hline wolffi+ grippotyphosa & 1 & 1.6 & $1 w$ & 0 & 0 & $1 g$ & 0 \\
\hline $\begin{array}{l}\text { icterohaemorrhagiae }+ \text { canicola }+ \\
\text { ballum }\end{array}$ & 1 & 1.6 & $1 c$ & 0 & $1 b$ & $1 i$ & 0 \\
\hline
\end{tabular}

\begin{tabular}{lll}
\hline Total & 63 & 100 \\
\hline
\end{tabular}

i: icterohaemorrhagie; b: ballum; p: pomona; g: grippotyphosa; w: wolffi; c: canicola

Cuadro 4. Animales serorreactores en una empresa ganadera de la Sierra Central y títulos de anticuerpos aglutinantes contra los serovares de Leptospira sp. en el grupo CONTROL (2003)

\begin{tabular}{|c|c|c|c|c|c|c|c|}
\hline \multirow[t]{2}{*}{ Leptospira sp } & \multicolumn{2}{|c|}{ Positivos } & \multicolumn{5}{|c|}{ Diluciones del suero } \\
\hline & $\mathrm{N}^{\mathrm{o}}$ & $\%$ & 100 & 200 & 400 & 800 & 3200 \\
\hline pomona & 0 & 0 & & & & & \\
\hline hardjo & 0 & 0 & & & & & \\
\hline canicola & 0 & 0 & & & & & \\
\hline icterohaemorrhagiae & 20 & 43.5 & 17 & 3 & & & \\
\hline grippotyphosa & 0 & 0 & & & & & \\
\hline wolffi & 0 & 0 & & & & & \\
\hline ballum & 20 & 43.5 & 9 & 6 & 5 & & \\
\hline bratislava & 0 & 0 & & & & & \\
\hline icterohaemorrhagiae + ballum & 6 & 13.0 & $3 i / 4 b$ & $2 i / 1 b$ & $1 i / 1 b$ & 0 & 0 \\
\hline Total & 46 & 100 & & & & & \\
\hline
\end{tabular}




\section{Agradecimientos}

Los autores agradecen al Dr. Máximo Gamarra Rojas por las valiosas informaciones sobre el sistema de manejo y problemas reproductivos de la población ovina de la SAIS en estudio. Asimismo, al Sr. Ricardo Ibáñez Zamudio por su excelente apoyo en la preparación de los antígenos de Leptospira sp. y en la prueba de MAT.

\section{Literatura Citada}

1. [AAVLD] Asociación Argentina de Veterinarios de Laboratorio de Diagnóstico. 1994. Manual de Leptospirosis. Buenos Aires, Argentina: AAVLD. 50 p.

2. Awad M, Willinger H. 1982. Evaluation of 2-mercapto-ethanol treatment in serodiagnosis of swine leptospirosis. Microbiologica 6: 133-143.

3. Barbudo Filho J, Girio RJS, Mathias LA, Oliveira AV, Marinho M. 1999. Pesquisa de anticorpos contra Leptospira interrogans em soros do ovinos do estado de São Paulo. Avaliacião do sorotipo jequitaia de Leptospira biflexa como antígeno de triagem sorológica. Ars Vet 15(1): 26-32.

4. Blackmore DK, Bahaman AR, Marshall RB. 1979. The epidemiological interpretation of serological responses to leptospiral serovars in sheep. NZ Vet J 30: 38-42.

5. Bolin C, Alt DP. 1998. Clinical signs, diagnosis, and prevention of bovine leptospirosis. Bovine Pract 33(1): 50-55.

6. Céspedes M, Llantoy L, Arizapana M, Unsihuay J. 2003. Seroprevalencia de leptospirosis en humanos, vacunos y perros en establos del valle del Mantaro. Rev Per Med Exp Salud Pública 20(3)S: S1-S3.

7. Ciceroni L, Bartoloni A, Pinto A, Guglielmetti P, Valdez C, Gamboa H, Roselli M, Giannico F, Paradisi F. 1997. Serological survey of leptospiral infections in sheep, goats and dogs in
Cordillera province, Bolivia. New Microbiol 20(1): 77-81.

8. Ciceroni L, Lombardo D, Pinto A, Ciarrocchi S, Simeoni J. 2000. Prevalence of antibodies to Leptospira in sheep and goats in Alto Adige-South Tyrol. J Vet Med 47: 217-223.

9. Collings DF. 1984. Leptospira interrogans infection in domestic and wild animals in Fiji. NZ Vet J 32: 21-24.

10. [DGIA] Dirección General de Información Agraria. 2002. Situación actual de la crianza de ovinos en el Perú. Ministerio de Agricultura. [Internet]. Disponible en: www.minag.gob.pe/ portalagrario

11. Hathaway S C, Little TW, Stevens AE. 1982. Serological survey of leptospiral antibodies in sheep from England and Wales. Vet Rec 110: 99-101.

12. Herrmann G, Pereira A, Moreira E, Haddad J, Rescende J, Rodríguez R, Leite $R$. 2004. Soroprêvalencia de aglutininas anti-Leptopsira sp. em ovinos nas mesorregiõnes sudeste e sudoeste do Estado Rio Grande do Sul, Brasil. Ciência Rural 34(2): 443-448.

13. [INEI] Instituto Nacional de Estadística e Informática. 1994. III Censo Nacional Agropecuario. [Internet]. Disponible en: http://www.inei.gob.pe

14. Levett P. 2001. Leptospirosis. Clin Microbiol Rev 14: 296-326.

15. Liceras de Hidalgo J, Valdivia S, Higushi E. 1989. Leptospirosis en el Perú. En: Anales del Seminario Nacional de Zoonosis y Enfermedades de Transmisión Alimentaria. Lima: MINSA. Programa Nacional de Control de Zoonosis. p 7-20.

16. Ochoa JE, Sánchez A, Ruiz I. 2000. Epidemiología de la leptospirosis en una zona andina de producción pecuaria. Rev Panam Salud Pública 7(5): 325-331.

17. [OIE] Organismo Internacional de Epizootias. 2006. Manual of diagnostic test and vaccines for terrestrial animals. Chapter 2.2.4. Leptospirosis. [Internet]. Disponible en: http://www.oie.int/ 
18. Quiroz JE. 1969. Encuesta serológica de leptospirosis en ovinos sacrificados en el camal de Yerbateros (Lima). Tesis Bachiller. Lima: Facultad de Medicina Veterinaria, Univ. Nacional Mayor de San Marcos. 35 p.

19. Rivera H. 2001. Causas frecuentes de aborto bovino. Rev Inv Vet, Perú 12(2):117-122.

20. Schoenian S. 2000. Infectious causes of abortion in ewes. Western Marylan Research. University of Maryland. [Internet]. Disponible en: www.sheepandgoat.com/articles/ abortion.html

21. Smits HL, Van Der Hoorn MA, Goris MG, Gussenhoven GC, Yersin C, Sasaki DM, Terpstra WJ, Hartskeerl
$\boldsymbol{R A}$. 2000. Simple latex agglutination for rapid serodiagnosis of human leptospirosis. J Clin Microbiol 38: 12721275.

22. Vanasco NB, Lottersberger J, Sequeira M, Tarabla H. 2001. Development and validation of an ELISA for the detection of leptospire-specific antibodies in rodents. Vet Microbiol 82: 321-330.

23. Vermunt J, West D, Arthur D, Marshall R. 1994. Leptospirosis in a lamb. NZ Vet J 42: 155.

24. Zamora J, Riedemann S, Tadish $N$. 1999. A serological survey of leptospirosis in sheep in Chile. Rev Latinoam Microbiol 41(2): 73-76. 